\title{
A Rare Occurrence of Sebaceous \\ Carcinoma, Sebaceoma, Syringocystadenoma Papilliferum, and Trichoblastoma in a Single Nevus Sebaceous Lesion
}

\author{
Amany Fathaddin ${ }^{a} \quad$ Eman Almukhadeb ${ }^{b}$ \\ aDepartment of Pathology, College of Medicine, King Saud University, King Khalid \\ University Hospital, Riyadh, Saudi Arabia; ${ }^{b}$ Department of Dermatology, College of \\ Medicine, King Saud University, King Khalid University Hospital, Riyadh, Saudi Arabia
}

\section{Keywords}

Nevus sebaceous · Sebaceoma - Sebaceous carcinoma - Syringosyctadenoma papilliferum .

Trichoblastoma

\section{Abstract}

Nevus sebaceous (NS) is a benign tumor with the potential to develop secondary benign and malignant neoplasms. It is a rare phenomenon to develop 2 or more skin tumors in a single NS lesion. We report a case of multiple secondary tumors, such as sebaceoma, sebaceous carcinoma, syringocystadenoma papilliferum, and trichoblastoma, in a single NS lesion.

\section{Introduction}

Nevus sebaceous (NS), also known as NS of Jadassohn or organoid nevus, was first described in 1895 by Jadassohn. It is a benign congenital hamartoma related to abnormalities of the epidermis, sebaceous glands, sweat glands, and hair follicles [1]. Clinically, it is usually apparent at birth as hairless patch. However, the lesion progresses and enlarges with age and typically presents as verrucous plaque at puberty. The most common sites of occurrence are the scalp and the face [2-4]. Mehregan and Pinkus in 1965 divided the natural progression of NS into 3 phases - infancy, puberty, and adulthood - and noticed that it is most likely to develop secondary neoplasms in the last stage [4]. NS occurs in $0.3 \%$ of newborns [5]. Several studies and case reports have confirmed that both benign and malignant tumors can develop in the 
setting of NS. Most of these, however, are benign. The studies also suggested delay of prophylactic surgical management to adolescence as malignant tumors are very rare in children $[2,6]$.

We describe a rare case of sebaceous carcinoma arising in combination with sebaceoma, trichoblastoma, and syringocystadenoma papilliferum (SCAP) in the setting of NS. To our knowledge, only a total of 30 cases of sebaceous carcinoma arising in NS have been reported in the literature [2, 6-9]. Only 5 of those were associated with 3 other neoplasms [7, 9].

\section{Case Report}

A 62-year-old female patient presented with the history of hairless patch over the right temporoparietal scalp since birth for which she never sought medical advice. She developed a mass on the top of this patch that had gradually enlarged over the last 6 months. Physical examination revealed a poorly defined $4 \times 3-\mathrm{cm}$, erythematous orange-colored plaque extending from the right temporoparietal scalp to the temporal face. On top of this plaque, there was a single $1.5 \times 1$-cm yellowish dome-shaped eroded nodule (Fig. 1). There were no palpable lymph nodes or organomegaly.

The initial incisional biopsy from the yellowish nodule showed a malignant basaloid tumor with focal sebaceous differentiation and was diagnosed as sebaceous carcinoma. On immunohistochemistry, MLH-1, MSH-2, MSH-6, and PMS-2 showed intact nuclear expression. Magnetic resonance imaging of the brain, bone scan, and computed tomography scan were done to rule out metastasis, and all were unremarkable.

The lesion was then completely excised with 1-cm margins and was sent for histopathology examination. Microscopic examination of the formalin-fixed, paraffin-embedded, and hematoxylin- and eosin-stained tissue sections from the ulcerated nodule showed irregular lobules in the dermis composed mainly of basophilic pleomorphic cells with little cytoplasm and prominent nucleoli (Fig. 2a). Admixed rare foci of differentiated sebaceous cells were noted (Fig. 2b). Comedo-type necrosis and a large number of mitoses were also seen (Fig. 2c). Immunohistochemical stains showed positive reaction to adipophilin (Fig. 2d) in the mature sebocytes and the tumor cells. EMA showed a positive reaction in the mature sebocytes. Ber-EP4 and CK20 showed negative reaction. The diagnosis of poorly differentiated sebaceous carcinoma was confirmed on this portion of the tissue. Around this nodule, another

Fig. 1. Poorly defined scarring alopecia plaques studded with yellowish papules, nodules, and crusted eroded blackish papules.

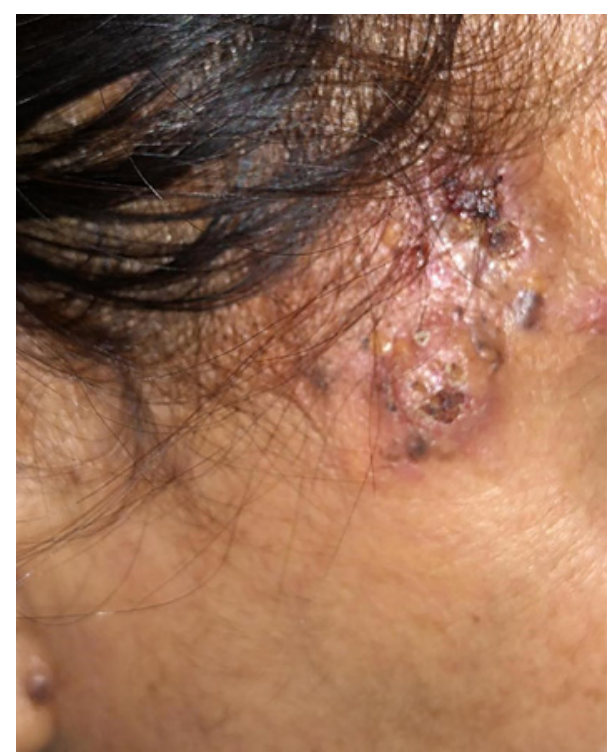




\section{Case Reports in Dermatology}
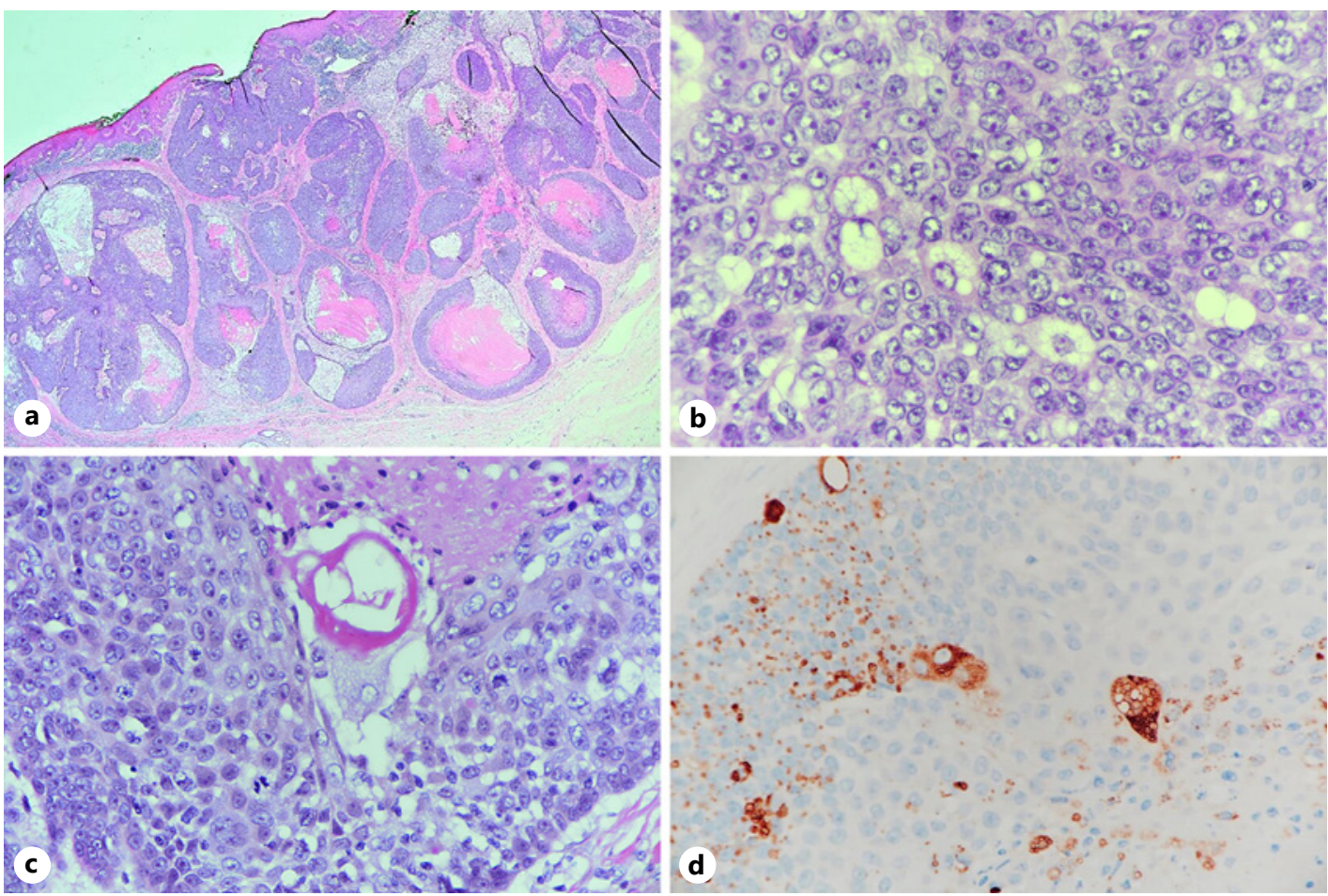

Fig. 2. a Sebaceous carcinoma irregular lobules of basaloid cells in the dermis $(H \& E . \times 20)$. b Mature sebocytes with vacuolated cytoplasm and scalloped nuclei (H\&E. $\times 400)$. c Tumor cells show nuclear atypia, and several mitotic figures and necrosis are also seen (H\&E. $\times 400)$. $\mathbf{d}$ Tumor cells showed positive reaction for adipophilin immunohistochemical stain $(\times 400)$.

Fig. 3. Trichoblastoma: benign basaloid tumor with no peripheral palisading, clefting, or sebaceous differentiation (H\&E. ×400).

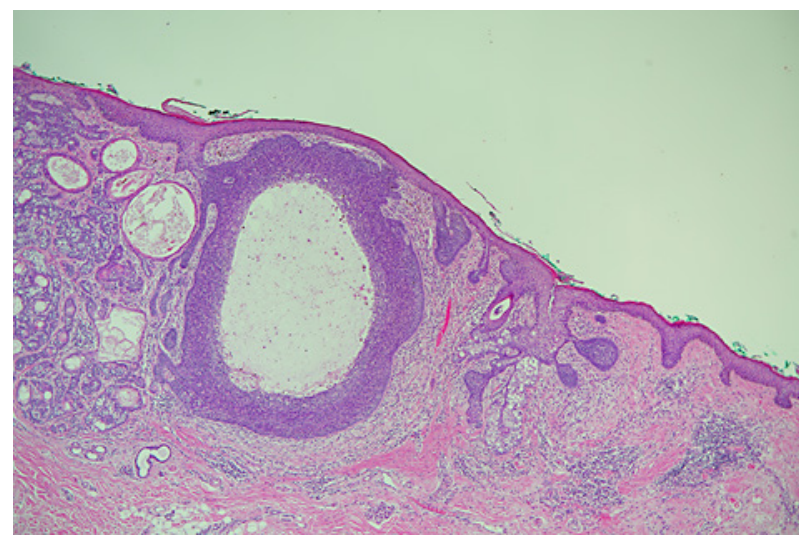

basaloid tumor with peripheral palisading was seen with no evidence of clefting between the stroma and the tumor cells, and this tumor was diagnosed as trichoblastoma (Fig. 3). Adjacent to trichoblastoma, there was another small superficial basaloid tumor with sebaceous differentiation but without peripheral palisading, mitoses, or necrosis, consistent with sebaceoma (Fig. 4). Examination of more sections showed an invagination of the epidermis filled with papillae communicating with duct-like structures in the deep aspect of the lesion (Fig. 5). These papillae were lined by an outer layer of tall columnar cells with the eosinophilic cytoplasm and an inner layer of cuboidal cells, and a diagnosis of SCAP was made for this lesion. The background skin showed hyperkeratosis, epidermal papillomatosis, and acanthosis. Several lobules of sebaceous glands communicated directly with the epidermal surface 


\section{Case Reports in Dermatology}

\begin{tabular}{l|l}
\hline Case Rep Dermatol 2021;13:271-277 \\
\hline DOI: 10.1159/000516351 & $\begin{array}{l}\text { C) 2021 The Author(s). Published by S. Karger AG, Basel } \\
\text { www.karger.com/cde }\end{array}$ \\
\hline
\end{tabular}

Fathaddin and Almukhadeb: A Rare Occurrence of Four Tumors in Single Nevus Sebaceous

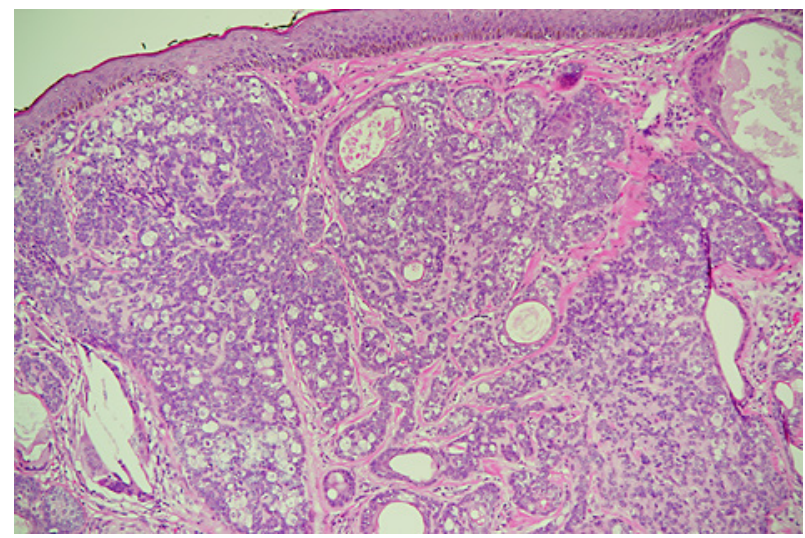

Fig. 4. Sebaceoma: benign basaloid tumor with sebaceous differentiation (H\&E. $\times 100)$.

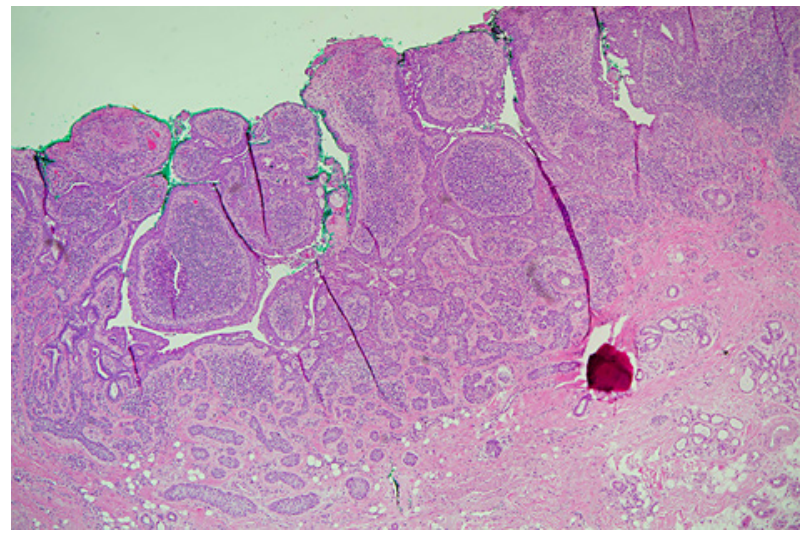

Fig. 5. SCAP: invagination of the epidermis filled with papillae lined by 2 cell layers (H\&E. $\times 40$ ). SCAP, syringocystadenoma papilliferum.

Fig. 6. NS in the background: epidermal hyperplasia with sebaceous glands opening directly to the surface (H\&E. $\times 40)$. NS, nevus sebaceous.

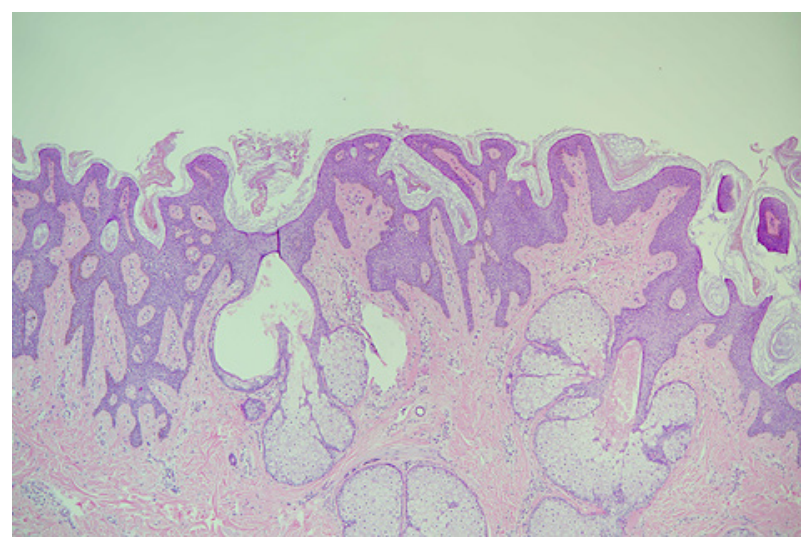

(Fig. 6). Apocrine glands were also seen in the deep dermis. Based on these findings, the final diagnosis was poorly differentiated as sebaceous carcinoma, sebaceoma, trichoblastoma, and SCAP in a NS background. Completeness of excision was confirmed.

\section{Discussion}

NS is a benign hamartoma, which is presents at birth, with the scalp being the most common location [1]. Histological findings vary with age, in infancy, and early childhood; the epidermis is relatively normal with immature pilosebaceous units. With age, the lesion 
Fathaddin and Almukhadeb: A Rare Occurrence of Four Tumors in Single Nevus Sebaceous

progresses and enlarges. During adolescence and adulthood, it shows hyperkeratosis and papillomatous epidermal hyperplasia. At this stage, the sebaceous glands are hyperplastic and open directly to the surface. Apocrine glands are also observed $[3,4,6]$. Secondary benign and malignant neoplasms have been described in association with NS. The largest retrospective study was published in 2013 by Idriss and Elston. It included 707 cases; the average age of the patients was 27.4 (range 0-95) years, and most of the nevi were located on the scalp. The adult stage was the most common stage biopsied. The study confirmed that NS is associated with secondary neoplasms that were seen in $21.4 \%$ of the cases, most of which were benign (18.9\%), while malignant tumors accounted for the rest $(2.5 \%)$. Malignant tumors occurred almost exclusively in adults [6]. Different numbers were reported in other studies ranging between 1.1 and 18\% for benign tumors and between 0.8 and $3.5 \%$ for malignant tumors $[2,6,10-12]$. The absence of malignant tumors was reported in some studies [13]. Idriss and Elston found trichoblastoma to be the most common benign tumor, while several other studies have reported SCAP as the most common benign tumor, followed by trichoblastoma $[2,10-12]$. Basal cell carcinoma has been reported as the most common malignant tumor in all the published studies [2, 6, 10-12].

Our case showed a combination of 4 tumors, both benign and malignant: SCAP, sebaceoma, trichoblastoma, and sebaceous carcinoma. Occurrence of 4 tumors in 1 case of NS is a rare event, with only 30 reported cases of sebaceous carcinoma arising in NS, and most of them were in combination with 2 other benign or malignant tumors. However, 5 of them occurred in combination with 3 or more other tumors [2, 6-9].

Sebaceous carcinoma commonly arises in the eyelid and less commonly shows extraocular locations. Sebaceous carcinoma arising in a clinical setting of NS is very unusual. Review of the previously published material showed that SC arising as a secondary neoplasm in NS had a female predilection, mostly located in the scalp with a tendency to occur in elderly patients (mean 67.7 years). The lesion clinically presented as an ulcerated nodule or mass. It was usually solitary and often with a recent history of rapid growth. It could occur alone or, more commonly, in combination with other benign and malignant adnexal tumors. None of the reported cases showed an association with Muir-Torre syndrome. Almost all the cases were treated with complete local excision only. Follow-up showed no evidence or recurrence, metastasis, or tumor-related death [7-9]. This is unlike the prognosis of the ocular sebaceous carcinoma, with less risk of recurrence and metastases [14].

Histologically, sebaceous carcinoma shows irregular nodules or sheets composed of basaloid cells with scanty cytoplasm and nuclei with variable degree of atypia. The extent of sebaceous differentiation, in the form of maturing sebocytes with vacuolated cytoplasm and scalloped nuclei, determines the tumor grade. Poorly differentiated tumors have less mature sebocytes than moderately and well-differentiated tumors. Mitoses including atypical ones are usually identified easily. Comedo-type necrosis is a common finding. Peripheral palisading and stromal cleft are not the features of sebaceous carcinoma [7-9]. Adipophilin immunohistochemical stain can highlight the lipid droplets in the tumor cell cytoplasm [8].

In summary, NS is a congenital hamartoma commonly located in the head and the neck area. The age of presentation and biopsy results are variable. The lesion has characteristic histopathological findings that can differentiate it from other cutaneous hamartomas. Secondary neoplasms from different lineages of differentiation can arise in association with NS. However, they are rare in children of different age-groups; thus, it is recommended to delay the prophylactic excision till puberty. Sebaceous carcinoma, although rare, can arise in lesions of NS. These are not associated with Muir-Torre syndrome, and de novo sebaceous carcinomas have a good prognosis.

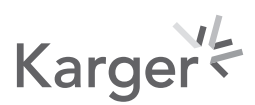




\section{Case Reports in Dermatology}

\begin{tabular}{l|l}
\hline Case Rep Dermatol 2021;13:271-277 \\
\hline DOI: 10.1159/000516351 & $\begin{array}{l}\text { @ 2021 The Author(s). Published by S. Karger AG, Basel } \\
\text { www.karger.com/cde }\end{array}$ \\
\hline
\end{tabular}

Fathaddin and Almukhadeb: A Rare Occurrence of Four Tumors in Single Nevus Sebaceous

\section{Acknowledgements}

This research is funded by the College of Medicine Research Center, deanship of Scientific Research, King Saud University.

\section{Statement of Ethics}

This case report was approved by the IRB Committee at the College of Medicine, King Saud University. Consent for patient photography was obtained. This case report complies with the guidelines for human studies.

The patients in this manuscript have given written informed consent for publication of their case details.

\section{Conflict of Interest Statement}

The authors have no conflicts of interest to declare.

\section{Funding Source}

This study was funded by the College of Medicine Research Center, deanship of scientific research, King Saud University.

\section{Author Contributions}

First author (A. Fathaddin) contributed in writing the pathological part, while the second author (E. Almukhadeb) contributed in writing the clinical part.

\section{References}

1 Pinkus H. Organoid nevus. Mod Probl Paediatr. 1976;20:50-7.

2 Hsu MC, Liau JY, Hong JL, Cheng Y, Liao YH, Chen JS, et al. Secondary neoplasms arising from nevus sebaceus: a retrospective study of 450 cases in Taiwan. J Dermatol. 2016;43:175-80.

3 Simi CM, Rajalakshmi T, Correa M. Clinicopathologic analysis of 21 cases of nevus sebaceus: a retrospective study. Indian J Dermatol Venereol Leprol. 2008;74:625-7.

4 Mehregan AH, Pinkus H. Life history of organoid nevi. Special reference to nevus sebaceus of Jadassohn. Arch Dermatol. 1965;91:574-88.

5 Alper J, Holmes LB, Mihm MC Jr. Birthmarks with serious medical significance: nevocullular nevi, sebaceous nevi, and multiple café au lait spotscance: nevocullular nevi, sebaceous nevi, and multiple cafe au lait spots. J Pediatr. 1979;95:696.

6 Idriss MH, Elston DM. Secondary neoplasms associated with nevus sebaceus of Jadassohn: a study of 707 cases. J Am Acad Dermatol. 2014;70:332-7.

7 Kazakov DV, Calonje E, Zelger B, Luzar B, Belousova IE, Mukensnabl P, et al. Sebaceous carcinoma arising in nevus sebaceus of Jadassohn: aclinicopathological study of five cases. Am J Dermatopathol. 2007;29:242-8.

8 Izumi M, Tang X, Chiu CS, Nagai T, Matsubayashi J, Iwaya K, et al. Ten cases of sebaceous carcinoma arising in nevus sebaceus. J Dermatol. 2008;35:704-11.

9 Wang E, Lee JS, Kazakov DV. A rare combination of sebaceoma with carcinomatous change (sebaceous carcinoma), trichoblastoma, and poroma arising from a nevus sebaceus. J Cutan Pathol. 2013;40:676-82.

10 Cribier B, Scrivener Y, Grosshans E. Tumors arising in nevus sebaceus: a study of 596 cases. J Am Acad Dermatol. 2000;42:263-8. 
11 Munoz-Perez MA, Garcia-Hernandez MJ, Rios JJ, Camacho F. Sebaceus naevi: a clinicopathologic study. J Eur Acad Dermatol Venereol. 2002;16:319-24.

12 Rosen H, Schmidt B, Lam HP, Meara JG, Labow BI. Management of nevus sebaceous and the risk of Basal cell carcinoma: an 18-year review. Pediatr Dermatol. 2009;26:676-81.

13 Santibanez-Gallerani A, Marshall D, Duarte A, Melnick SJ, Thaller S. Should nevus sebaceus of Jadassohn in children be excised? A study of 757 cases, and literature review. J Craniofac Surg. 2003;14:658-60.

14 Patterson JW, Wick MR. Tumor and tumor-like conditions with predominantly sebaceous differentiation. Nonmelanocytic tumors of the skin. AFIP atlas of tumor pathology. Washington, DC: ARP Press; 2006. p. 117-134. 